\title{
rhlL-1Ra reduces hepatocellular apoptosis in mice with acetaminophen-induced acute liver failure
}

\author{
Jianjun $\mathrm{Hu}^{1}$, Dejun $\mathrm{Yan}^{2,3}$, Jin Gao ${ }^{4}$, Chuanying $\mathrm{Xu}^{2}$, Yunsheng Yuan ${ }^{2}$, Runzhi Zhu ${ }^{2}$, Di Xiang ${ }^{4}$, Shunyan Weng ${ }^{5}$, \\ Wei $\mathrm{Han}^{4}$, Guoqing Zang ${ }^{1, *}$ and Yan $\mathrm{Yu}^{2, *}$
}

Acute liver failure (ALF) is a life-threatening disease that has proven difficult to cure. In Western countries, acetaminophen (APAP) poisoning is the most common cause of ALF. However, the mode of cell death in APAP-induced ALF cases is controversial. Previous studies have shown that administration of anti-interleukin-1 (anti-IL-1) antibody attenuated APAP-induced liver injury, and that administration of anti-IL-1 receptor antagonist (anti-IL-1Ra) antibody exacerbated organ injury. These results prompted us to investigate the roles of IL-1Ra in APAP-induced ALF mice. Our results show that administration of recombinant human IL-1Ra (rhIL-1Ra) could significantly improve the survival rate of mice with ALF induced by APAP. Furthermore, we found that rhIL-1Ras could dramatically inhibit the activities of alanine aminotransferase and aspartate aminotransferase in serum, reduce the death of hepatocytes and accelerate the proliferation of hepatocytes. In addition, we show that hepatocellular apoptosis rather than necrosis was the major cause of ALF-induced animal death, and that the anti-apoptosis role of rhIL-1Ra was mediated by reducing the release of cytochrome $c$ from the mitochondria, and the activities of caspase-3, caspase- 8 and caspase-9 in the liver tissue. In conclusion, these data indicate that rhIL-1Ra is a promising candidate for the treatment of APAP-induced ALF in mice through the reduction of hepatocellular apoptosis.

Laboratory Investigation (2010) 90, 1737-1746; doi:10.1038/labinvest.2010.127; published online 19 July 2010

KEYWORDS: acetaminophen; acute liver failure; apoptosis; hepatotoxicity; interleukin-1 receptor antagonist

Acute liver failure (ALF) is a life-threatening disease marked by the sudden loss of hepatic function without previous history of liver disease, the massive death of hepatocytes and only minor liver regeneration. ${ }^{1,2}$ The mode of cell death in acetaminophen (APAP)-induced ALF cases is still controversial. However, many recent studies have suggested that hepatocyte apoptosis has a more important role than hepatocyte necrosis in liver failure. ${ }^{3-6}$ To date, APAP is the most common etiology of ALF in Western countries. ${ }^{7} \mathrm{~N}$-acetyl-Lcysteine, which can restore the glutathione pool depleted by the APAP-derived major toxic metabolite, $N$-acetyl- $p$-benzoquinone imine (NAPQI), can be used in the clinical treatment of ALF induced by APAP, but is effective only when administered before an advanced stage of ALF. ${ }^{8,9}$ For pathogen-induced ALF, there is still no effective therapy, except for emergent liver transplantation. ${ }^{10}$ However, the application of this technology is greatly limited because of limited sources of liver donors and the expensive cost of treatment. Consequently, it is important to determine whether there is effective drug therapy for the treatment of ALF.

The interleukin (IL)- 1 family consists of 11 members, including 2 agonists, namely IL- $1 \alpha$ and IL- $1 \beta$, two receptors, namely biologically active IL-1RI and inert IL-1RII, as well as a specific receptor antagonist, ie, IL-1 receptor antagonist (IL-1Ra). IL-1Ra is structurally similar to IL-1, which can bind solidly to the IL-1 receptor, and thus blocks the IL-1/IL1RI signal pathway.

Both IL-1 and IL-1Ra are produced abundantly by various kinds of cells such as neutrophils, macrophages and fibroblasts in various patients with inflammatory conditions. ${ }^{11}$

\footnotetext{
${ }^{1}$ Department of Infectious Disease, Shanghai No. 6 People's Hospital, School of Medicine, Shanghai Jiao Tong University, Shanghai, China; ${ }^{2}$ Shanghai Municipality Key Laboratory of Animal Biotechnology, School of Agriculture and Biology, Shanghai Jiao Tong University, Shanghai, China; ${ }^{3}$ Department of Biological Sciences, Bowling Green State University, Bowling Green, $\mathrm{OH}$, USA; ${ }^{4}$ Laboratory of Regeneromics, School of Pharmacy, Shanghai Jiao Tong University, Shanghai, China and ${ }^{5}$ School of Life Science and Biotechnology, Shanghai Jiao Tong University, Shanghai, China

Correspondence: Professor Y Yu, PhD, Shanghai Municipality Key Laboratory of Animal Biotechnology, School of Agriculture and Biology, Shanghai Jiao Tong University, Shanghai 200240, China. E-mail: yanyu@sjtu.edu.cn and Professor G Zang, PhD, Department of Infectious Disease, Shanghai No. 6 People's Hospital, School of Medicine, Shanghai Jiao Tong University, Shanghai 200233, China. E-mail: zangguoqin@126.com
}

*The authors equally contributed to this work.

Received 16 February 2010; revised 14 April 2010; accepted 23 April 2010 
At present, it is known that expressions of IL-1 and IL-1Ra in tissue are enhanced significantly and that the intrahepatic IL-1 level is correlated with the severity of organ damage in APAP-induced liver injury. ${ }^{12-15}$ Blazka et al showed that the administration of anti-IL-1 antibody attenuated APAP-induced liver injury and that the administration of anti-IL-1Ra antibody exacerbated organ injury. However, IL-1Ra $(4 \mathrm{ml} / \mathrm{kg})$ that was administered $30 \mathrm{~min}$ before APAP had only a modest protective effect against APAP-induced liver injury on decreasing serum enzyme release, and had no effect on the degree of hepatic congestion or necrosis. ${ }^{16,17}$ Thus, we hypothesized that some suitable dose of recombinant human IL-1Ra (rhIL-1Ra) could effectively attenuate hepatotoxicity in APAP-induced ALF mice.

In this study, we investigated the biological activity of rhIL-1Ra in APAP-induced ALF mouse and the mode of cell death during ALF.

\section{MATERIALS AND METHODS Reagents and Antibodies}

The following reagents and antibodies were used in this study: APAP (A7085-100G, Sigma-Aldrich, St Louis, MO, USA), In Situ Cell Death Detection Kit (Roche, Mannheim, Germany), rabbit anti-mouse proliferating cell nuclear antigen (PCNA) mAb (sc-25280, Santa Cruz Biochemistry, Santa Cruz, CA, USA), Mitochondria/Cytosol Fractionation Kit (Biovision, CA, USA), caspase-3, caspase- 8 and caspase-9 colorimetric assay kits (Biovison), rabbit anti-mouse Cytochrome $c \mathrm{mAb}$ (no. 4280, Cell Signaling Technology, Boston, MA, USA), rabbit anti-mouse Bax antibody (50599-2-Ig, ProteinTech, IL, USA), mouse $\beta$-actin mAb (sc-47778, Santa Cruz Biochemistry) and secondary antibody (goat anti-rabbit pAbs and rabbit anti-mouse pAbs, Boster, Wuhan, China). rhIL-1Ra was expressed and purified in Escherichia coli in our laboratory, with protein purity of $98 \%$ and endotoxin content of no more than $0.2 \mathrm{EU} / \mu \mathrm{g}$. The specific activity of the protein was testified in vitro.

\section{Animals}

Wild-type (WT) male C57BL/6 mice, aged 8-10 weeks, were purchased from the Shanghai Laboratory Animal Center (Shanghai, China). All animals were maintained under standard conditions and received humane care in accordance with the protocols approved by the Shanghai Jiao Tong University and with the legal requirements in China. Mice were allowed to adapt to their environment for 1 week before initiating the experiments. Room temperature was maintained at $25^{\circ} \mathrm{C}$ during the animal experiments.

\section{Animal Experiments}

APAP was temporarily diluted in sterile phosphate-buffered saline right before use and rhIL-1Ra in sterile normal saline (NS), with the concentration of 15 and $0.25 \mathrm{mg} / \mathrm{ml}$, respectively.
In the first set of experiments, APAP-induced ALF was achieved in all mice, by a single intraperitoneal injection with 650 or $550 \mathrm{mg} / \mathrm{kg}$ of APAP. One hour later, mice in the rhIL$1 \mathrm{Ra}$ groups were treated by subcutaneous injection with $1 \mathrm{mg} / \mathrm{kg}$ weight of rhIL-1Ra per $12 \mathrm{~h}$ till $168 \mathrm{~h}$ after APAP injection, and mice in the NS groups were treated with NS as control. The number of mice that died in every group was recorded after APAP injection.

In the next set of experiments, all mice were intoxicated with $550 \mathrm{mg} / \mathrm{kg}$ of APAP, followed by treatments with rhIL$1 \mathrm{Ra}$ or NS as described above. Whole-blood samples were collected after APAP injection to determine serum activities of ALT and AST using a Fuji DRI-CHEM $3500 \mathrm{~V}$ as instructed by the manufacturer (Fuji Medical System, Tokyo, Japan), and the livers were collected for biochemical (snap frozen in liquid nitrogen and stored at $-80^{\circ} \mathrm{C}$ until use) and histological studies (fixed in $4 \%$ formaldehyde solution for $24 \mathrm{~h}$ ) at $3,6,12,24,48,96$ and $168 \mathrm{~h}$ time points. Tissue samples were uniformly separated from the same part of the left lobe and the right middle lobe of the livers.

\section{Histological Examination Liver}

Liver samples were cut into small pieces $\left(<0.1 \mathrm{~cm}^{3}\right)$, fixed in formaldehyde solution, dehydrated, paraffin embedded and cut at $5-\mu \mathrm{m}$ thickness. Sections were stained with hematoxylin-eosin. Tdt-mediated dUTP-x nick-end labeling staining was performed using the In Situ Cell Death Detection Kit. PCNA was determined by immunohistochemistry. The analysis of histological examination was performed by NISElements Basic Research (Nikon, Kanagawa, Japan). Two sections were adopted from the left and right middle lobes in every liver, and eight low-magnification views were captured for calculation in each section.

\section{Cytochrome $c$ and Bax Determination by Western Blotting}

Liver samples were homogenized with PIPA buffer (Beyotime, Jiangsu, China), and the concentration of proteins in each lysate was detected by the BSA microbiuret assay (Beyotime). Proteins were extracted using the Mitochondria/ Cytosol Fractionation Kit. Cytochrome $c$ determination was performed on cytosolic fraction (S100) and on enriched mitochondria fraction (HM) of liver extracts, and Bax and $\beta$-actin determination was performed on liver lysates by western blotting. Proteins were electrophoresed on a SDS-PAGE gel (4\% stacking gel and 15\% running gel), and then transferred to PVDF membranes (Pall, NY, USA). The membranes were then hybridized by turns with primary and secondary antibodies. Signal detection was performed by using enhanced chemiluminescence reagents (Thermo, IL, USA).

\section{Evaluation of Caspase-3, Caspase-8 and Caspase-9 Activity}

Caspase-3, caspase- 8 and caspase- 9 activities were detected in liver extracts using caspase-3, caspase- 8 and caspase- 9 
colorimetric assay kits, respectively. Enzymatic activities of the three caspases in liver extracts from mice treated only by NS were also detected as controls. The kits were used as recommended by the manufacturer.

\section{Statistical Analysis}

The statistical significance of difference between treatment groups and control groups was analyzed by unpaired $t$-test for necrosis area of the liver, the number of apoptotic cells, the number of PCNA-positive cells and the activities of caspases between two groups. The Kaplan-Meier method was used for comparing survival rate, and ANOVA was used when involving three or more groups. $P<0.05$ was accepted as significant.

\section{RESULTS}

\section{rhIL-1Ra Significantly Improved the Survival Rate of Mice Treated with APAP}

After $48 \mathrm{~h}$ of APAP injection, the survival rate of mice intoxicated with $650 \mathrm{mg} / \mathrm{kg}$ of APAP in the rhIL-1Ra group was significantly higher than that of the NS group (80 vs 33.33\%, $P<0.05$ ) (Figure 1a). Similar results were obtained in
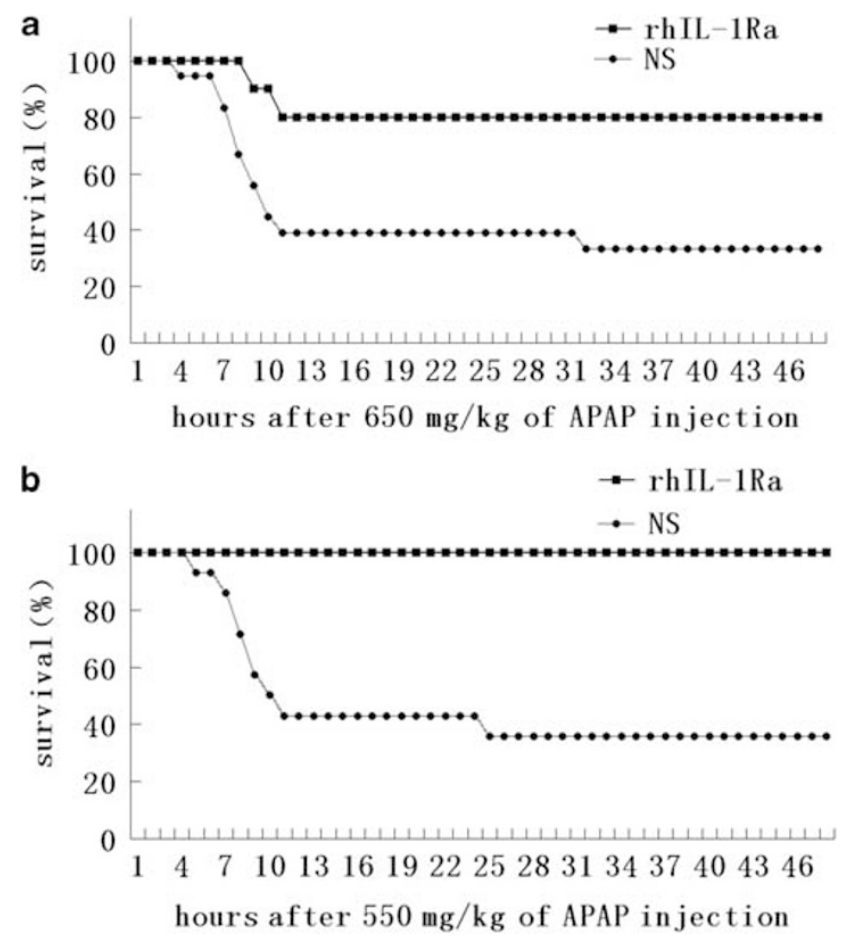

Figure 1 rhIL-1Ra dramatically improved survival rates in ALF mice intoxicated with either 650 or $550 \mathrm{mg} / \mathrm{kg}$ APAP. Survival rates were compared only over a period of $48 \mathrm{~h}$ (no further deaths occurred), and only two results out of the four experiments are shown herein. (a) Intoxicated with $650 \mathrm{mg} / \mathrm{kg}$ APAP, the survival rate of mice in the rhlL-1Ra group ( $n=10)$ was significantly improved compared with that in the NS group $(n=18)(P<0.05)$. (b) Intoxicated with $550 \mathrm{mg} / \mathrm{kg}$ APAP, the survival rate of mice in the rhlL-1Ra group $(n=14)$ was very significantly improved compared with that in the NS group $(n=14)(P<0.01)$. mice after they were intoxicated with $550 \mathrm{mg} / \mathrm{kg}$ of APAP (100 vs $35.71 \%, P<0.01$ ) (Figure 1b). Both experiments were repeated. The numbers of animals and conditions in replicate experiments were identical to the two experiments; and similar results were obtained (detailed data not shown).

In 4 experiments, 46 ( 6 in the rhIL-1Ra groups and 40 in the NS groups) out of 112 mice (48 in the rhIL-1Ra groups and 64 in the NS groups) died, and all deaths occurred within $48 \mathrm{~h}$ after APAP injection. Most of the deaths (91.3\%, 42 of 46) occurred within $12 \mathrm{~h}$, of which $82.61 \%$ (38 of 46 ) occurred between 6 and $12 \mathrm{~h}$. These results were similar to those data reported in other literatures studying the survival rate of ALF mice induced by APAP. ${ }^{18-20}$

\section{rhIL-1Ra Inhibited the Activities of Serum Enzymes}

Three and six hours after treatment with $550 \mathrm{mg} / \mathrm{kg}$ of APAP, serum ALT and AST activities were significantly increased in the NS and rhIL-1Ra groups compared with the control groups. However, activities of the two serum enzymes were significantly inhibited in the rhIL-1Ra groups compared with the NS groups (Figure 2). At $3 \mathrm{~h}$ and $6 \mathrm{~h}$ time points, ALT activities in the rhIL-1Ra groups were reduced by 67.78 and $82.28 \%$, respectively (Figure 2a) $(P<0.01 v s$ NS groups), and AST activities were reduced by 62.85 and $78.99 \%$, respectively (Figure $2 \mathrm{~b})(P<0.01 v s$ NS groups).
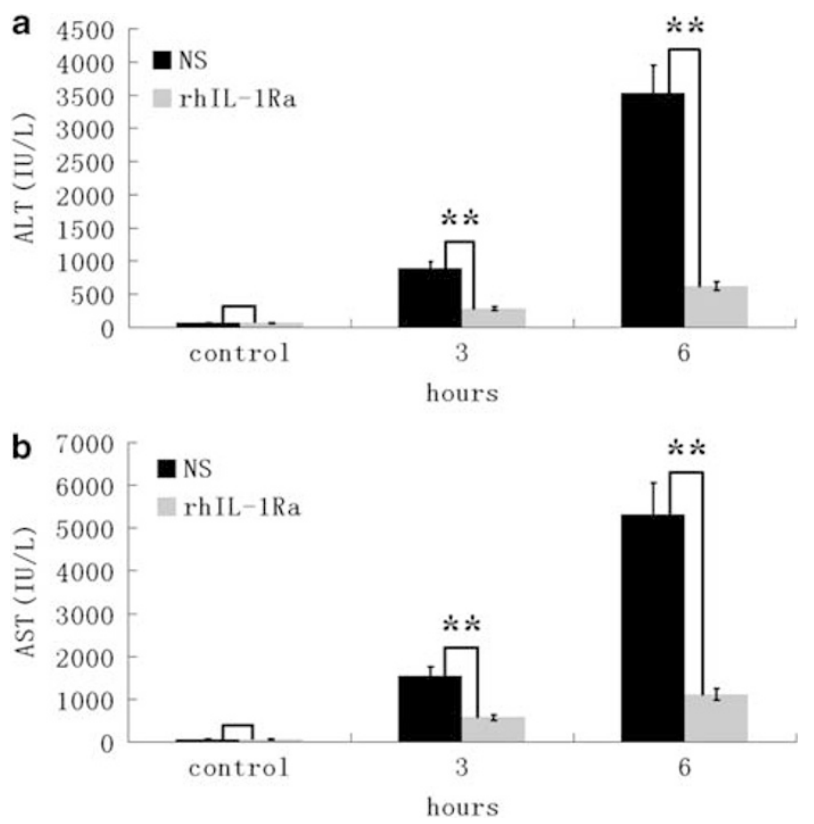

Figure 2 rhIL-1Ra significantly inhibited the activities of ALT and AST in serum. (a) The serum ALT activities in the rhIL-1Ra groups were significantly inhibited compared with that of the NS groups at 3 and $6 \mathrm{~h}$ time points ( $n=10$ per time point, $\left.{ }^{* *} P<0.01\right)$. (b) The serum AST activities in the rhlL1 Ra groups were significantly inhibited compared with that of the NS groups at 3 and $6 \mathrm{~h}$ time points $\left({ }^{*} P<0.01\right)$. 


\section{rhIL-1Ra Reduced the Death of Hepatocytes after APAP Induction}

The livers of control mice treated with NS or rhIL-1Ra alone were morphologically normal (photo not shown). Three and six hours after intoxication by APAP, the livers displayed obvious confluent necrosis, present in a centrizonal (mainly zone 3 , some zone 2) necrosis pattern accompanied with little or no inflammation, with some steatosis in residual viable hepatocytes (Figure $3 \mathrm{~b}$ ). In general, the lesions were more limited in the rhIL-1Ra groups than in the NS groups, and the necrosis area was significantly smaller in the rhIL-1Ra groups than in the NS groups (Figure 3c). rhIL-1Ra also exerts its beneficial effect by strongly inhibiting APAP-induced apoptosis of hepatocytes (Figure 4 ) at 3 and $6 \mathrm{~h}$ time points.

\section{rhIL-1Ra Improved the Survival Rate of ALF Mice Mainly by Inhibiting Severe Apoptosis of Hepatocytes}

Interestingly, those mice that died from ALF did not display the most severe hepatocyte necrosis; instead, they presented the most hepatocyte apoptosis when compared with the surviving mice at all time points (data detailed not shown). In fact, the surviving mice displayed the most sever necrosis and only minor apoptosis of hepatocytes at the $48 \mathrm{~h}$ time point (Figure 5). However, no deaths were recorded at this time point. These results suggest that severe apoptosis of hepatocytes is more important than massive necrosis of hepatocytes as a cause of ALF, and that rhIL-1Ra significantly improved the survival rate of ALF mice primarily by efficiently inhibiting the apoptosis of hepatocytes.

\section{rhIL-1Ra Accelerated Hepatocyte Proliferation in ALF Mice}

Usually, the liver has the ability to regenerate after injury. However, liver regeneration was significantly inhibited in ALF patients who died when compared with survivors. ${ }^{2}$ It prompted us to investigate the effects of rhIL-1Ra on hepatocyte proliferation in ALF mice by PCNA detection. The results showed that more PCNA-positive hepatocytes were found in the rhIL-1Ra groups than in the NS groups at 3 and a

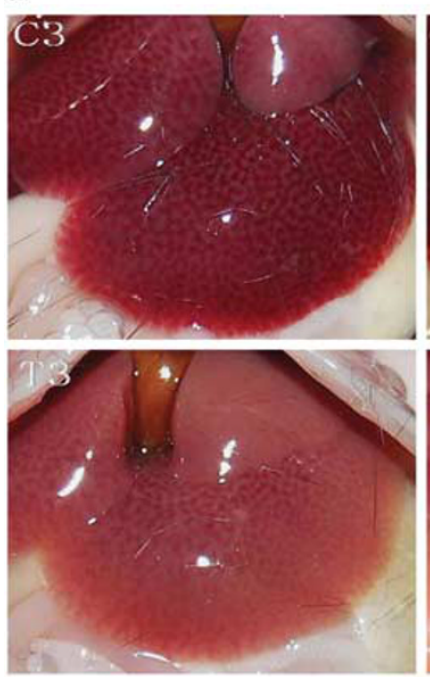

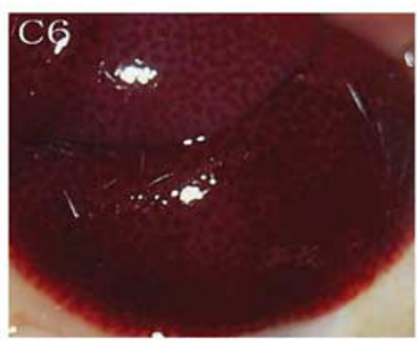

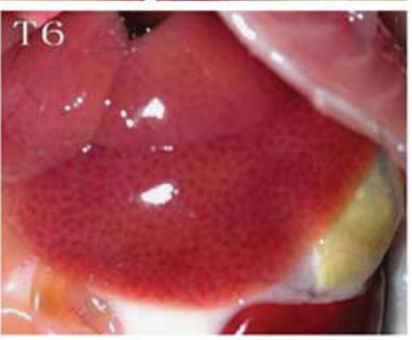

b
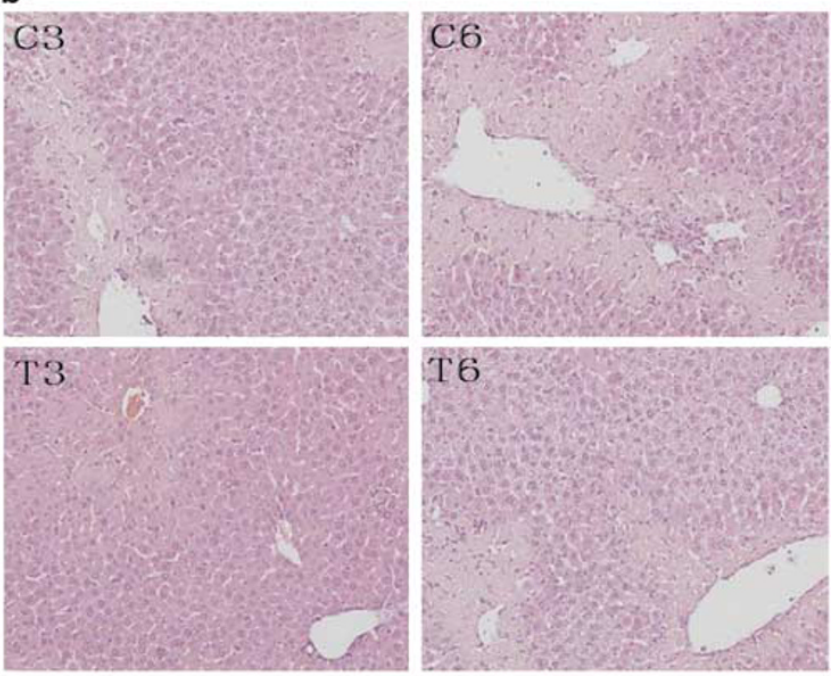

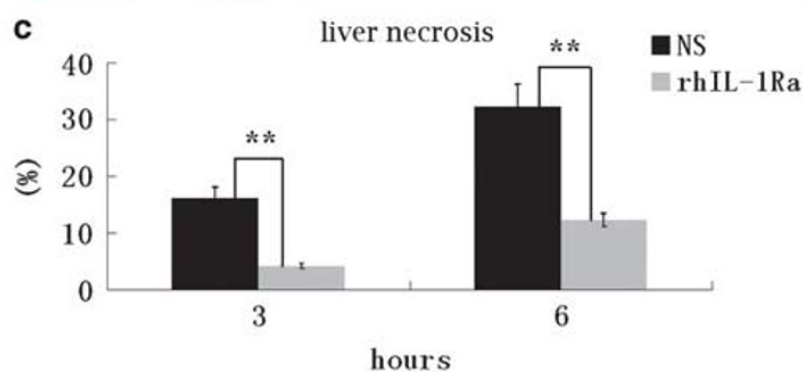

Figure 3 rhlL-1Ra significantly decreased necrosis area of liver in ALF mice intoxicated with $550 \mathrm{mg} / \mathrm{kg}$ of APAP. (a) Macroscopic views of the livers (original magnification). C3 and C6 represent the NS groups at 3 and $6 \mathrm{~h}$ time points, respectively, and T3 and T6 represent the rhIL-1Ra groups at 3 and $6 \mathrm{~h}$ time points, respectively. The livers of both rhlL-1Ra groups and NS groups appeared obvious swollen and sorrel compared with normal livers. However, the pathological changes of NS groups were more serious than that of rhIL-1Ra groups at 3 and $6 \mathrm{~h}$ time points. (b) Microscopic views of the livers (HE staining, original magnification $\times 100)$. (c) Necrosis area of the liver in the rhlL-1Ra groups was significantly decreased compared with that of the NS groups at 3 and $6 \mathrm{~h}$ time points $\left({ }^{* * P}<0.01\right)$. 
a
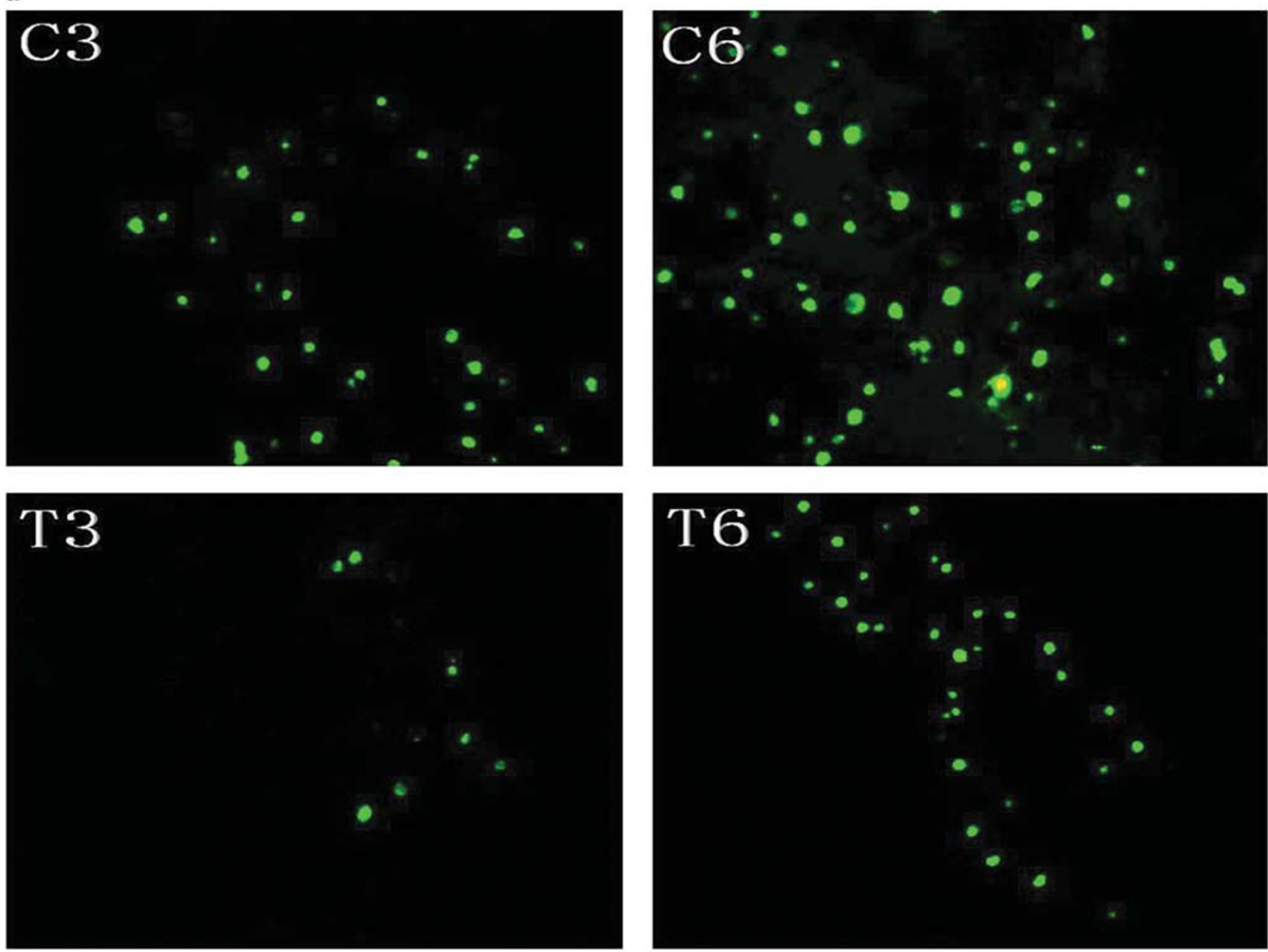

b

apoptotic cells

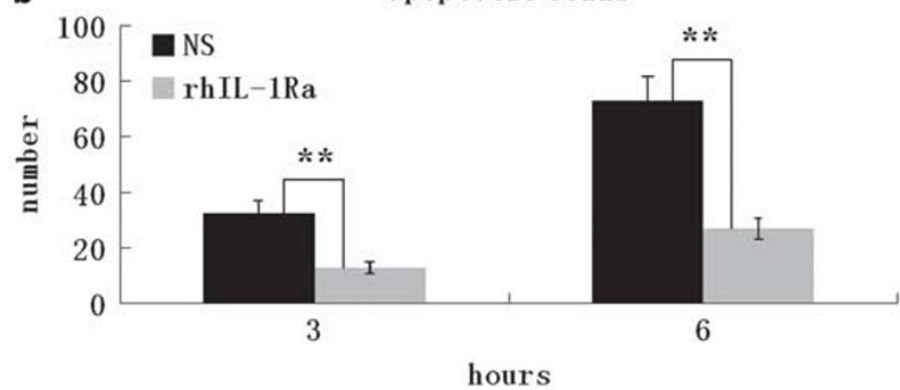

Figure 4 rhlL-1Ra significantly protected hepatocytes from apoptosis in ALF mice intoxicated with $550 \mathrm{mg} / \mathrm{kg}$ of APAP. (a) DNA fragmentation in the livers of mice (TUNEL staining, original magnification $\times 200$ ). C3 and C6 represent the NS groups at 3 and $6 \mathrm{~h}$ time points, respectively, and T3 and T6 represent the rhIL-1Ra groups at 3 and $6 \mathrm{~h}$ time points, respectively. (b)The number of positive cells in the livers from the rhIL-1Ra groups was significantly decreased compared with that of the NS groups at 3 and $6 \mathrm{~h}$ time points $(* * P<0.01)$. The numbers were expressed per view $(\times 200)$.

$6 \mathrm{~h}$ time points (Figure 6). We also investigated the effects of rhIL-1Ra on hepatocyte proliferation in mice that recovered from ALF, and found that rhIL-1Ra significantly accelerated the progress of cell proliferation starting $24 \mathrm{~h}$ after APAP intoxication (Figure 7). These results suggested that rhIL-1Ra could efficiently accelerate the proliferation of hepatocytes both in the stage of progression and recovery in ALF induced by APAP.

\section{rhIL-1Ra Effectively Reduced the Release of Cytochrome $c$ from the Mitochondria and Inhibited Caspase Activities}

After displaying the effect of rhIL-1Ra on protecting hepatocytes from apoptosis induced by APAP, we investigated the relationship between rhIL-1Ra injection and the cascade of apoptosis pathways. As shown (in Figure 8a), the release of cytochrome $c$ and Bax expression in the rhIL-1Ra groups was 
a
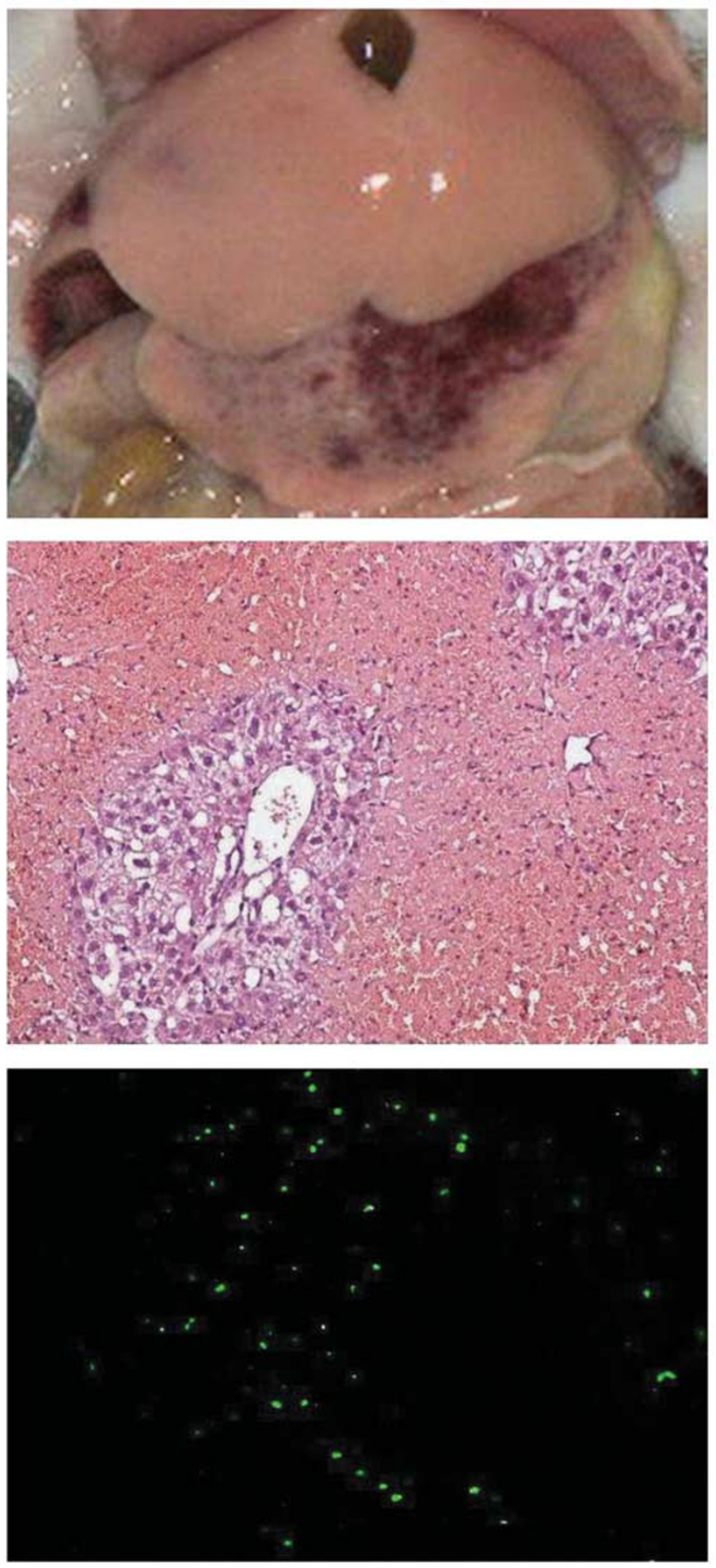

b
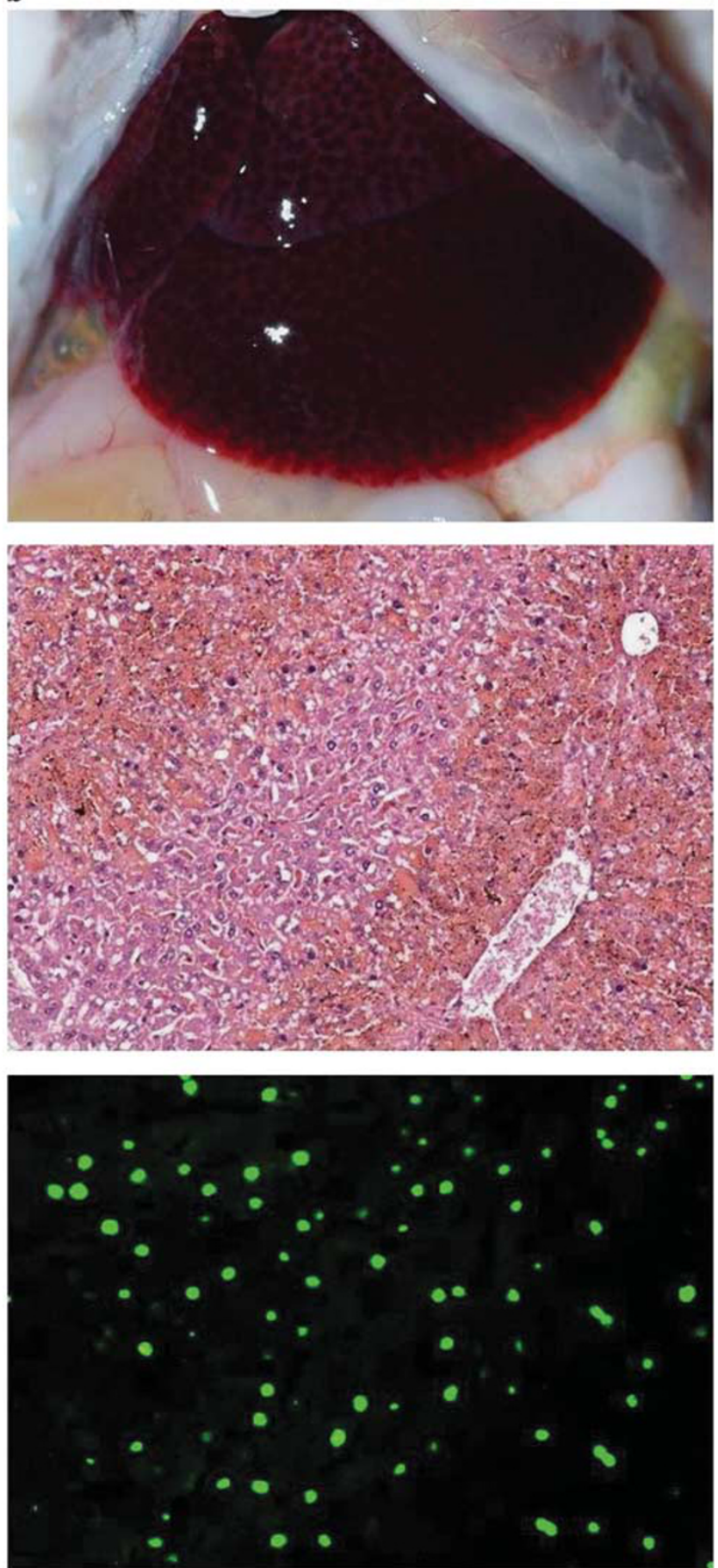

Figure 5 Comparison between the livers of mice that recovered from ALF induced by $550 \mathrm{mg} / \mathrm{kg}$ of APAP and those that died. (a) Photographs of the livers from surviving mice at $48 \mathrm{~h}$ time point $(n=5)$. (Upper) macroscopic views of the livers. The livers appeared to be yellow and swollen with obvious lesion. (Middle) microscopic views of the livers (HE staining, original magnification $\times 100$ ). Mice displayed the most serious necrosis of livers. (Bottom) DNA fragmentation in the livers (TUNEL staining, original magnification $\times 200$ ) Only minor apoptosis of hepatocytes was detected. (b) Photographs of the livers from mice that could not survive from $\operatorname{ALF}(n=26)$, and the liver samples were harvested within 2 min after death. (Upper) macroscopic views of the livers. The livers appeared to be deeply mahogany and swollen. (Middle) microscopic views of the livers. Mice displayed obvious necrosis with some cell autolysis. (Bottom) DNA fragmentation in the livers. The most serious apoptosis of hepatocytes was detected in the mice.

significantly inhibited compared with those in the NS groups at 3 and $6 \mathrm{~h}$ time points. Similarly, at 3 and $6 \mathrm{~h}$ time points, the activities of caspase- 3 , caspase- 8 and caspase- 9 in the liver were significantly lower in the rhIL-1Ra groups than those in the NS groups (Figure $8 \mathrm{~b}-\mathrm{d}$ ). These data indicate that rhIL1 Ra efficiently reduced hepatocyte apoptosis after APAP 

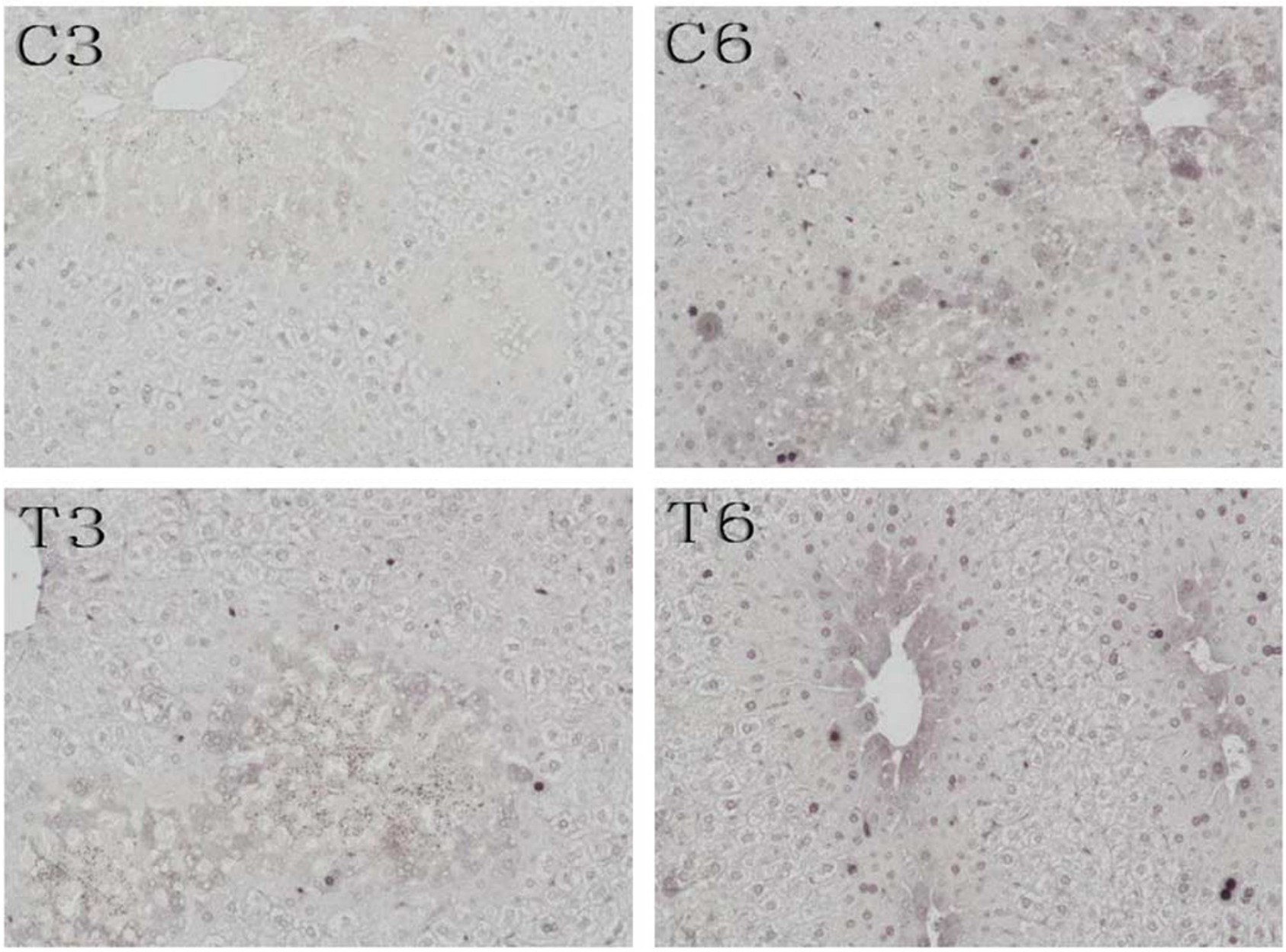

b

\section{PCNA-positive ce11s}

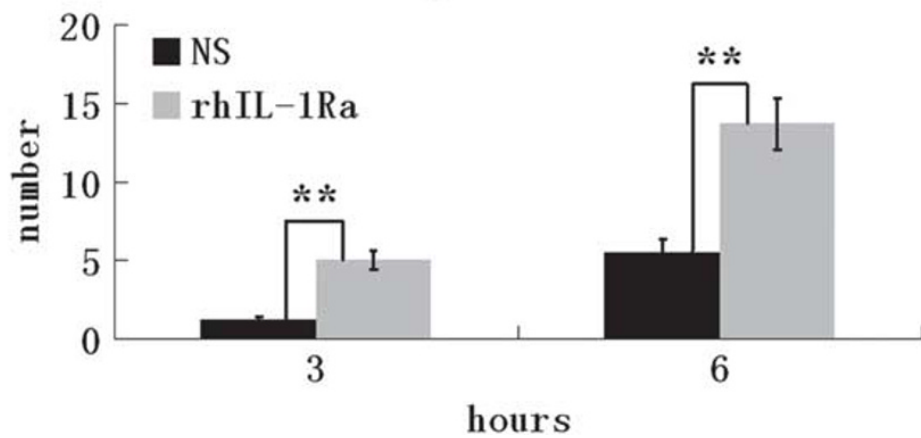

Figure 6 rhIL-1Ra significantly accelerated the proliferation of hepatocytes in ALF mice intoxicated with $550 \mathrm{mg} / \mathrm{kg}$ of APAP. (a) A small number of PCNA-positive cells can be detected at 3 and $6 \mathrm{~h}$ time points (immunohistology, original magnification $\times 100$ ). C3 and C6 represent the NS groups at 3 and $6 \mathrm{~h}$ time points, respectively, and T3 and T6 represent the rhIL-1Ra groups at 3 and $6 \mathrm{~h}$ time points, respectively. (b) The number of PCNA-positive cells in the rhIL-1Ra groups was significantly increased when comparing with the NS groups at 3 and $6 \mathrm{~h}$ time points $(* * P<0.01)$. The numbers were expressed per view $(\times 100)$.

induction by inhibiting the activities of cytochrome $c$ and caspases-mediated apoptosis pathways.

\section{DISCUSSION}

In 2001, rhIL-1Ra was approved to be used in clinic to treat patients with refractory rheumatoid arthritis, to whom conventional drugs had shown very limited effects. Blocking IL-1 activity with IL-1Ra results in an effective interruption in the progressive joint space narrowing. ${ }^{11}$ Many studies have shown that IL-1Ra exhibits powerful roles in antagonizing inflammation. ${ }^{21,22}$ Fujioka et $a l^{23}$ reported that the sequential treatment with killed Propioniobacterium acnes 
a
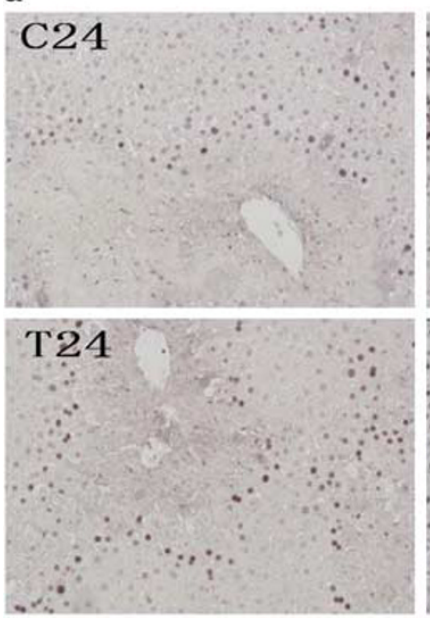
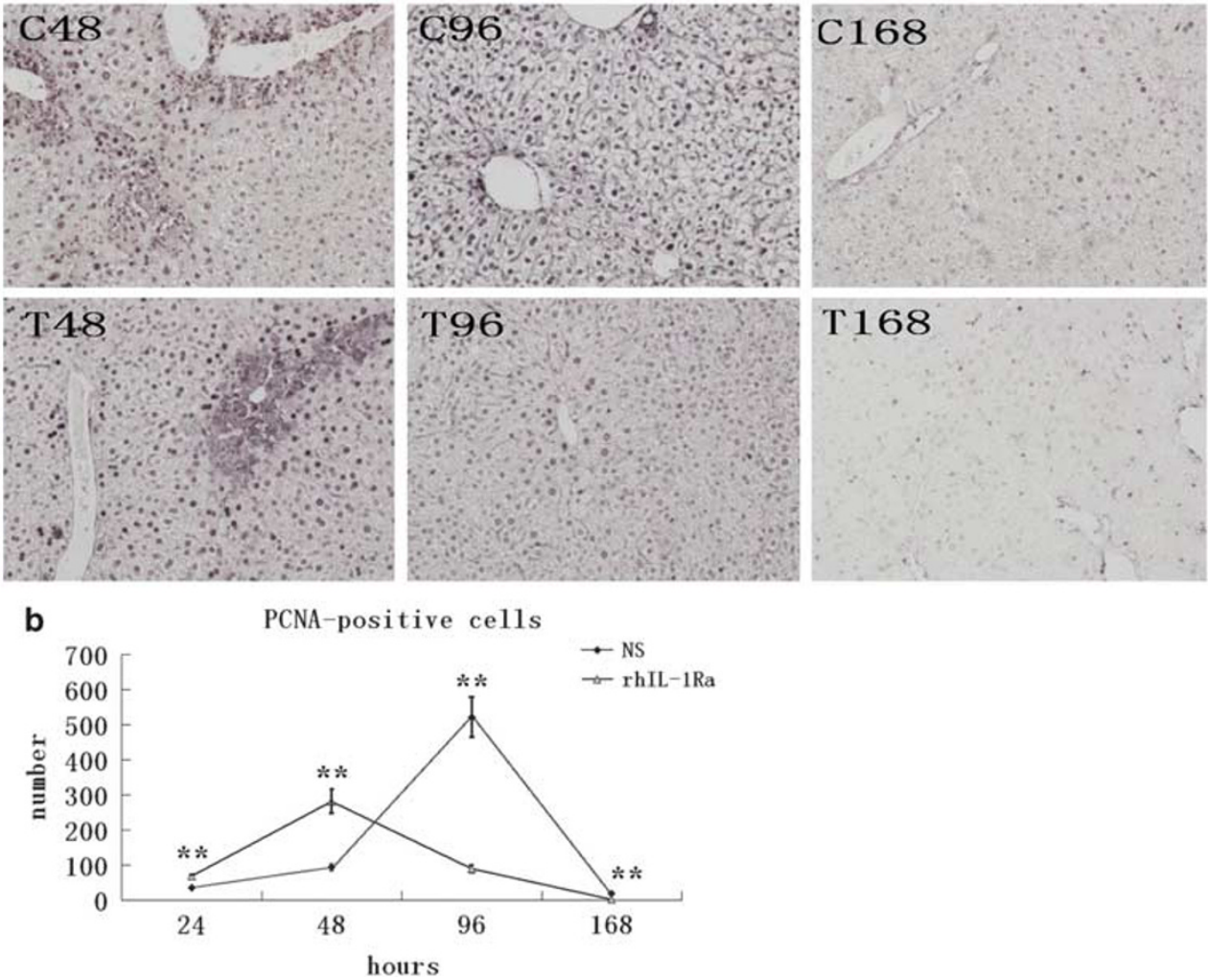

Figure $7 \mathrm{rhlL}-1$ Ra significantly accelerated the proliferation of hepatocytes in mice that recovered from ALF induced by $550 \mathrm{mg} / \mathrm{kg}$ of APAP after $24 \mathrm{~h}$ time point. (a) A large number of PCNA-positive cells can be detected at 24, 48 and $96 \mathrm{~h}$ time points (original magnification $\times 100$ ). C24, C48, C96 and C168 represent the NS groups at $24,48,96$ and $168 \mathrm{~h}$ time points ( $n=4-6$ per time point), respectively, and T24, T48, T96 and T168 represent the rhIL-1Ra groups at $24,48,96$ and $168 \mathrm{~h}$ time points ( $n=8-10$ per time point), respectively. (b) The number of PCNA-positive cells in the rhIL-1Ra groups was significantly larger than that of the NS groups at 24 and $48 \mathrm{~h}$ time points $\left({ }^{*} P<0.01\right)$, and the peak number occurred at $48 \mathrm{~h}$ in the rhIL-1Ra groups, while occurring at $96 \mathrm{~h}$ in the NS groups. The numbers were expressed per view $(\times 100)$.
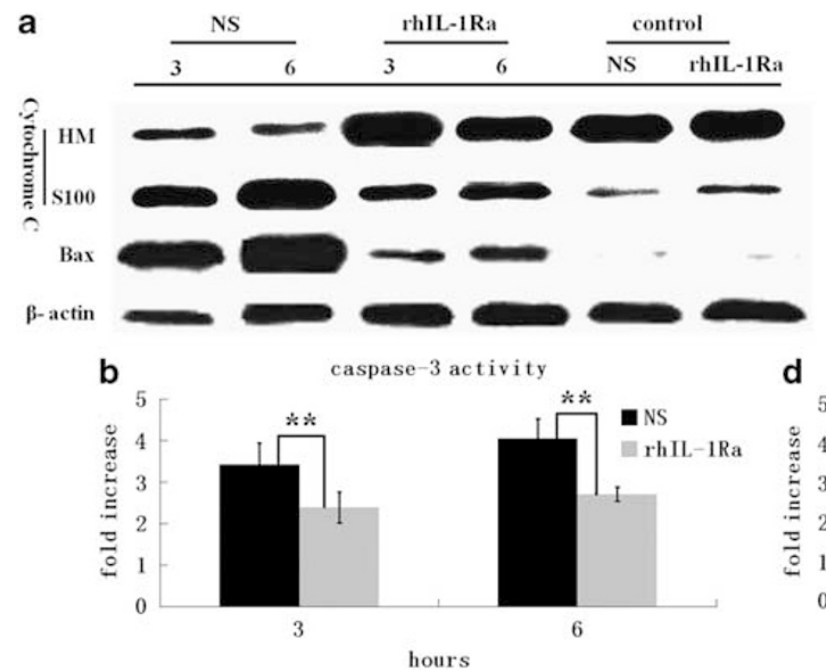

c

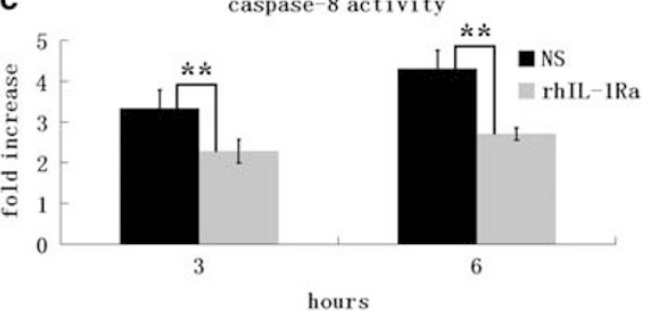

d

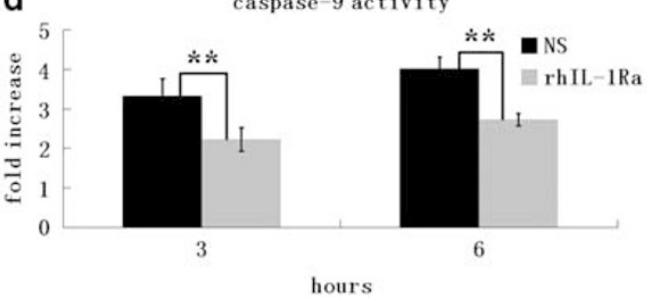

Figure 8 Effects of rhlL-1Ra on apoptosis pathways of hepatocytes in ALF mice intoxicated with $550 \mathrm{mg} / \mathrm{kg}$ of APAP. (a) At 3 and $6 \mathrm{~h}$ time points, the release of cytochrome $c$ from the mitochondria (HM) into the cytosol (S100) and Bax expression in the rhlL-1Ra groups was significantly inhibited compared with those of the NS groups. $\beta$-Actin levels were shown as a control. One of three experiments with similar results is shown. (b-d) At 3 and $6 \mathrm{~h}$ time points, activities of caspase-3, caspase- 8 and caspase- 9 in the liver were significantly lower in mice of the rhIL-1Ra groups than in those of the NS groups $(* * P<0.01)$. 
and lipopolysaccharide caused hepatic necrosis, along with enhanced intrahepatic expression of IL-1ra and IL-1, and that the neutralization of IL-1ra further exacerbated liver injury. Blazka et $a l^{16,17}$ displayed similar results in APAP-induced liver injury mice. However, when mice were administered IL$1 \mathrm{Ra}(4 \mathrm{ml} / \mathrm{kg})$, they observed only a modest protective effect on decreasing serum enzyme release and no effect on histopathological changes. It prompts us to investigate whether a suitable dose of rhIL-1Ra could effectively attenuate hepatotoxicity in APAP-induced ALF mice.

In our previous study, we performed some experiments to investigate the dose-effect relationship of rhIL-1Ra in APAP-induced ALF mice (unpublished data). Eventually, we chose $1 \mathrm{mg} / \mathrm{kg}$ weight of rhIL-1Ra to accomplish further studies. We demonstrated that $1 \mathrm{mg} / \mathrm{kg}$ of rhIL-1Ra significantly improved the survival rate of ALF mice induced by either 650 or $550 \mathrm{mg} / \mathrm{kg}$ of APAP, inhibited the activities of ALT and AST, reduced the necrosis and apoptosis of hepatocytes and accelerated the proliferation of hepatocytes in ALF mice induced by $550 \mathrm{mg} / \mathrm{kg}$ of APAP. The study also suggests that serious hepatocyte apoptosis rather than massive necrosis is the major cause resulting in the death of ALF mice, and that the effect of rhIL-1Ra is mediated mainly by strongly inhibiting apoptosis pathways, including reducing the release of cytochrome $c$ from the mitochondria, inhibiting Bax expression and decreasing the activities of caspase- 3 , caspase- 8 and caspase- 9 in the liver tissue.

It may be very possible that the role of rhIL-1Ra on promoting cell proliferation results from reducing tissue damage. To further investigate the role of IL-1Ra in involving in the network regulating liver regeneration, we treated SD rats with a single subcutaneous injection of rabbit polyclonal antibody against rhIL-1Ra in another study, followed by $70 \%$ partial hepatectomy $(\mathrm{PH})$ half an hour after the injection, and found that the antibody significantly inhibited the recovery of liver mass relative to control on 2 and 4 days after $\mathrm{PH}$ (unpublished data). It suggested that IL-1Ra had a critical role in the network.

In a study, Ishibe et al found that IL-1ra-deficient (IL-1ra $\mathrm{KO}$ )-derived hepatocytes exhibited more resistance against APAP but not APAP-derived major toxic metabolite, NAPQI, compared with WT mouse-derived hepatocytes. Furthermore, the amounts of a major APAP adduct (seleniumbinding protein), an indicator of NAPQI generation from APAP, were also found to be significantly lower in IL-1ra KO mice than in WT mice along with depressed intrahepatic expression of cytochrome P450 enzymes (such as CYP1A2, CYP2E1 and CYP3A11), the enzymes crucially involved in NAPQI generation from APAP. ${ }^{12}$ These observations showed that IL-1ra deficiency impaired APAP metabolism and suggested that rhIL-1Ra would not attenuate the hepatotoxicity of APAP. On the contrary, it may even exacerbate hepatotoxicity. Thus, we could conclude that IL-1ra exhibited its protection character mainly by reducing apoptosis of hepa- tocytes rather than by interfering with the APAP metabolism. However, an investigation on the interaction between rhIL1 Ra and APAP is still very important to clarify what effects rhIL-1Ra has on APAP metabolism.

Some studies have shown that moderate hypothermia may affect liver injury ${ }^{24}$ or hepatic regeneration ${ }^{25}$ in ALF mice and in humans. ${ }^{26}$ Thus, to take temperature into consideration, we maintained all mice at a $25^{\circ} \mathrm{C}$ environment to ensure constant body temperature of mice as much as possible.

In conclusion, the present data suggest that serious hepatocyte apoptosis is the major cause resulting in death of APAP-induced ALF mice and that rhIL-1Ra can be further researched and developed as a candidate for the treatment of the disease mainly based on its effect on reducing hepatocellular apoptosis.

\section{ACKNOWLEDGEMENT}

We thank the Chinese National 863 Project (2007AA02Z100) for providing financial support to the study.

\section{DISCLOSURE/CONFLICT OF INTEREST}

The authors declare no conflict of interest.

1. Malhi H, Gores GJ, Lemasters JJ. Apoptosis and necrosis in the liver: a tale of two deaths? Hepatology 2006;43(S1):S31-S44.

2. Rutherford A, Chung RT. Acute liver failure: mechanisms of hepatocyte injury and regeneration. Semin Liver Dis 2008;28:167-174.

3. Cohen SD, Khairallah EA. Selective protein arylation and acetaminophen-induced hepatotoxicity. Drug Metab Rev 1997;29: 59-77.

4. Gujral JS, Knight TR, Farhood A, et al. Mode of cell death after acetaminophen overdose in mice: apoptosis or oncotic necrosis? Toxicol Sci 2002;67:322-328.

5. Kon K, Ikejima K, Okumura K, et al. Role of apoptosis in acetaminophen hepatotoxicity. J Gastroenterol Hepatol 2007;22(Suppl 1):S49-S52.

6. Ray SD, Kamendulis LM, Gurule MW, et al. Ca ${ }^{2+}$ antagonists inhibit DNA fragmentation and toxic cell death induced by acetaminophen. FASEB J 1993;7:453-463.

7. Bower WA, Johns M, Margolis HS, et al. Population-based surveillance for acute liver failure. Am J Gastroenterol 2007;102: 2459-2463.

8. Schiodt FV, Rochling FA, Casey DL, et al. Acetaminophen toxicity in an urban county hospital. N Engl J Med 1997;337:1112-1118.

9. Jones $A L$. Mechanism of action and value of $\mathrm{N}$-acetylcysteine in the treatment of early and late acetaminophen poisoning: a critical review. Clin Toxicol 1998;36:277-285.

10. Lee WM, Squires Jr RH, Nyberg SL, et al. Acute liver failure: summary of a workshop. Hepatology 2008;47:1401-1415.

11. Dinarello CA. Immunological and inflammatory functions of the interleukin-1 family. Annu Rev Immunol 2009;27:519-550.

12. Ishibe T, Kimura A, Ishida $Y$, et al. Reduced acetaminophen-induced liver injury in mice by genetic disruption of IL-1 receptor antagonist. Lab Invest 2009;89:68-79.

13. Ishida $Y$, Kondo $T$, Kimura A, et al. Opposite roles of neutrophils and macrophages in the pathogenesis of acetaminophen-induced acute liver injury. Eur J Immunol 2006;36:1028-1038.

14. Ishida $\mathrm{Y}$, Kondo $\mathrm{T}$, Tsuneyama K, et al. The pathogenic roles of tumor necrosis factor receptor p55 in acetaminophen-induced liver injury in mice. J Leukoc Biol 2004;75:59-67.

15. Ishida $\mathrm{Y}$, Kondo $\mathrm{T}$, Ohshima $\mathrm{T}$, et al. A pivotal involvement of IFN-gamma in the pathogenesis of acetaminophen-induced acute liver injury. FASEB J 2002;16:1227-1236.

16. Blazka ME, Elwell MR, Holladay SD, et al. Histopathology of acetaminophen-induced liver changes: role of interleukin 1 alpha and tumor necrosis factor alpha. Toxicol Pathol 1996;24:181-189. 
17. Blazka ME, Wilmer JL, Holladay SD, et al. Role of proinflammatory cytokines in acetaminophen hepatotoxicity. Toxicol Appl Pharmaco 1995; 133:43-52.

18. Ferret PJ, Hammoud R, Tulliez $M$, et al. Detoxification of reactive oxygen species by a nonpeptidyl mimic of superoxide dismutase cures acetaminophen-induced acute liver failure in the mouse. Hepatology 2001;33:1173-1180.

19. Bedda S, Laurent A, Conti F, et al. Mangafodipir prevents liver injury induced by acetaminophen in the mouse. J Hepatology 2003;39:765-772.

20. Lieu HT, Batteux F, Simon MT, et al. HIP/PAP accelerates liver regeneration and protects against acetaminophen injury in mice. Hepatology 2005;42:618-626.

21. Aksentijevich I, Masters SL, Ferguson PJ, et al. An autoinflammatory disease with deficiency of the interleukin-1-receptor antagonist. N Engl J Med 2009;360:2426-2437.
22. Reddy $S$, Jia $S$, Geoffrey $R$, et al. An autoinflammatory disease due to homozygous deletion of the IL1RN locus. N Engl J Med 2009;360: 2438-2444.

23. Fujioka N, Mukaida N, Harada A, et al. Preparation of specific antibodies against murine IL-1ra and the establishment of IL-1ra as an endogenous regulator of bacteria-induced fulminant hepatitis in mice. J Leukoc Biol 1995;58:90-98.

24. Vaquero J, Bélanger $M$, James $L$, et al. Mild hypothermia attenuates liver injury and improves survival in mice with acetaminophen toxicity. Gastroenterology 2007;132:372-383.

25. Munoz SJ. Hypothermia may impair hepatic regeneration in acute liver failure. Gastroenterology 2005;128:1143-1144.

26. Jalan R, Olde Damink SWM, Deutz NEP, et al. Moderate hypothermia in patients with acute liver failure and uncontrolled intracranial hypertension. Gastroenterology 2004;127:1338-1346. 